\title{
REGISTRO DE NIDIFICACIÓN DE LONCHURA MALACCA (AVES: ESTRILDIDAE) EN UN ECOSISTEMA AGRÍCOLA DE MAYABEQUE, CUBA
}

\section{Record of a nesting colony of Lonchura malacca (Aves: Estrildidae) in an agroecosystem of Mayabeque, Cuba}

\author{
Jorge Luis Guerra Solana ${ }^{1, *}$ y Duniel Barrios Valdés ${ }^{2}$
}

${ }^{1}$ División de Colecciones Zoológicas, Instituto de Ecología y Sistemática, CITMA. Carretera de Varona No. 11835, entre Oriente y Lindero, Reparto Parajón, Municipio Boyeros, La Habana 19, C.P. 11900, Cuba; (D) orcid.org/0000-00021318-4932. 'Jardín Botánico Nacional, Universidad de La Habana. Carretera El Rocío km 31⁄2, Calabazar, Boyeros, La Habana, C.P. 19230, Cuba; (D) orcid.org/0000-0002-7876-7645. *Para correspondencia: guerra@ecologia.cu.

\section{RESUMEN}

Se documenta la nidificación de la Monjita Tricolor, Lonchura malacca (Linnaeus, 1766), en un ecosistema agrícola de Mayabeque, Cuba. El registro tuvo lugar en un campo de caña de azúcar, dos kilómetros al este del pueblo de Quivicán. En este cultivo se ubicaron nueve nidos de Monjita, de los cuales uno contenía huevos y pichones recién eclosionados, y cuatro sólo huevos frescos. Las características de estos nidos, así como su contenido, son descritos.

Palabras clave: Lonchura malacca, nidificación, cultivo de caña, Cuba.

\section{ABSTRACT}

A nesting colony of Tricolored Munia, Lonchura malacca (Linnaeus, 1766), is reported here, located in an agroecosystem of Mayabeque, Cuba. The nesting area was located in a sugarcane farm two kilometers east of the town of Quivicán. Nine nests were found, and one of them had eggs and hatchling; four nests had only fresh eggs. Here we characterize the nests and describe the contents.

Keywords: Tricolored Munia, nesting record, sugarcane farm, Cuba.

La Monjita Tricolor (Lonchura malacca) es un estríldido nativo de la India y Sri Lanka, donde habita en pastizales, matorrales rodeados de vegetación secundaria y humedales. Esta especie se alimenta principalmente de granos, y es considerada en algunas regiones como plaga de cultivos agrícolas (Avery, 1979; Reidinger y Libay, 1979; Payne, 2018).

Hasta la fecha, la monjita se ha naturalizado en gran parte del Caribe, América Central y Sudamérica, así como Europa y norte de Asia (Sharpe et al., 1997; Otero-Vázquez, 1998; Lever, 2005; de Juana, 2006). Está evaluada por la IUCN como una especie de preocupación menor, debido a su amplia distribución y tamaños poblacionales estables (BirdLife International, 2016).

En las Antillas, la especie ha sido reportada en La Española, Puerto Rico, Jamaica y Martinica (Raffaele et al., 2003; Latta et al., 2006). En Cuba se registra por primera vez en 1991, y actualmente se le puede encontrar en toda la isla y cayos adyacentes, siendo abundante en algunas localidades (Kirkconnell y Garrido, 1992; Garrido y Kirkconnell, 2011; Rodríguez et al., 2014). 
Pese a que su presencia en Cuba es conocida desde hace más de dos décadas, la especie ha sido muy poco estudiada en el país, y solo existen referencias a su impacto negativo como plaga de cultivos de arroz y otras gramíneas (Acosta y Mugica, 2006; Saucedo et al., 2017). Hasta la fecha, no existen datos sobre su reproducción u otros aspectos de su historia de vida. Con el objetivo de llenar parte de los vacíos de información existentes, en este trabajo se aportan datos de una colonia de nidificación ubicada en un ecosistema agrícola de Quivicán, Mayabeque.

E1 15 de agosto de 2015, una colonia reproductiva de Monjita Tricolor fue localizada en el interior de una parcela de caña de azúcar de tres hectáreas, dos kilómetros al este del pueblo de Quivicán, Mayabeque (Fig. 1). La plantación tenía entre cinco y seis meses de sembrada y no sobrepasaba los dos metros de altura (Fig. 2A). La colonia era de nueve nidos (Tabla I), de los cuales uno contenía tres huevos y dos pichones recién eclosionados (Fig. 2D), y cuatro solo huevos frescos (1-6 huevos por nido). Los nidos estaban construidos de ramitas de hierba de guinea (Panicum maximum Jacq.) y cortadera (Cyperus sp.), recubiertos con hojas verdes de caña de azúcar (Fig. 2 B, C). La distancia entre los nidos no superaba los dos metros y todos se encontraban sobre las cañas (Saccharum officinarum), prácticamente a la misma altura $(1-1.5 \mathrm{~m})$ del suelo. El tamaño promedio de los nidos fue $17.5 \pm 3.0 \mathrm{~cm} \mathrm{x} 10.8 \pm 4.4 \mathrm{~cm}$ (diámetro mayor y menor), con un orificio de entrada de 4-6 cm en su parte más ancha. Los huevos fueron elípticos, blancos y de textura lisa. El tamaño promedio de estos fue de $17.9 \pm 0.7 \mathrm{~mm} \times 11.9 \pm 0.5 \mathrm{~mm}$ (diámetros mayor y menor), (Tabla II).

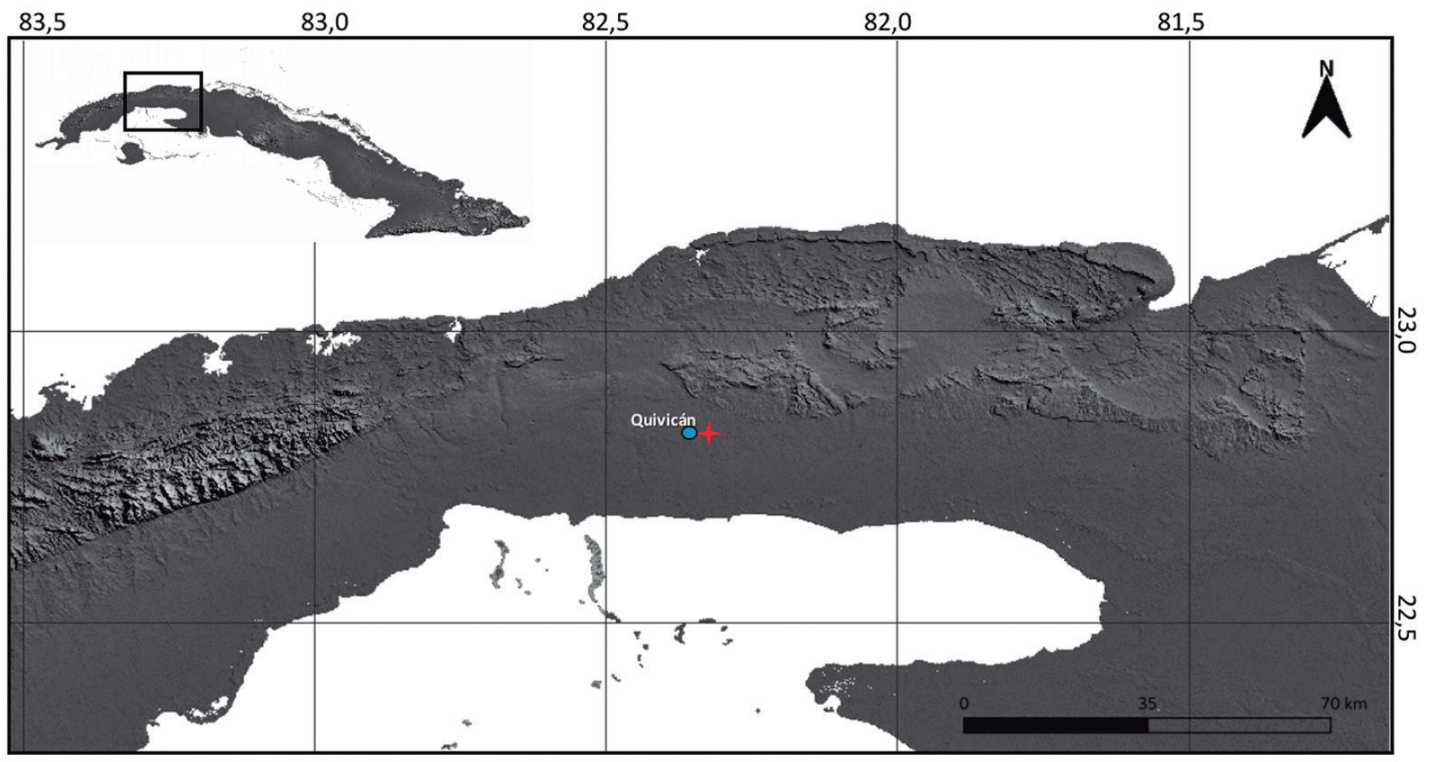

Figura 1. Ubicación geográfica de una colonia reproductiva de Lonchura malacca registrada en un cañaveral de Quivicán, Cuba, en agosto de 2015 . 


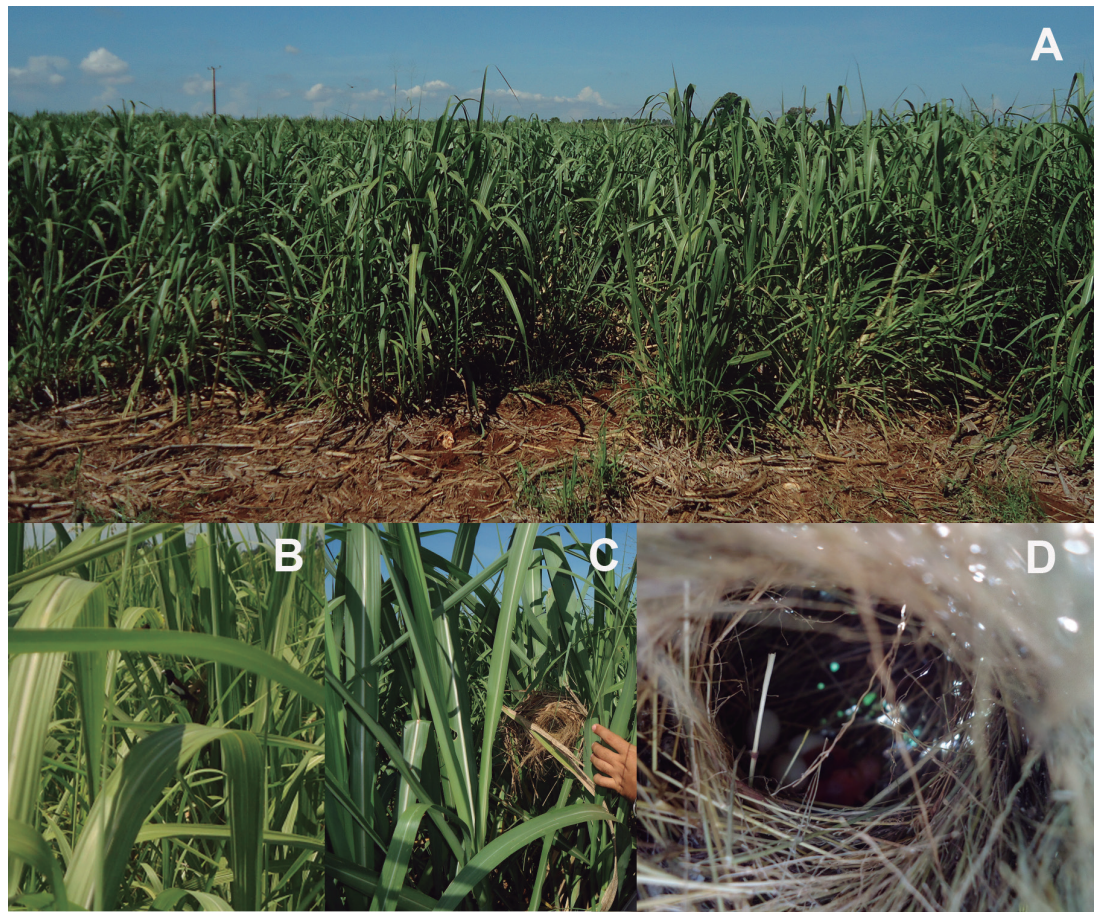

Figura 2. Sitio de nidificación de una colonia reproductiva de Lonchura malacca registrada en Quivicán, Cuba, en agosto de 2015. A, campo de caña de no más de seis meses de sembrado. B, adulto a la entrada del nido. C, nido. D, nido con huevos y pichones recién eclosionados.

Tabla I. Características de los nidos de una colonia de Lonchura malacca registrada en un cañaveral de Quivicán, Cuba, en agosto de 2015

\begin{tabular}{|c|c|c|c|c|}
\hline \multirow{2}{*}{ Nido } & \multirow{2}{*}{$\begin{array}{c}\text { Altura del } \\
\text { nido (cm) }\end{array}$} & \multicolumn{2}{|c|}{ Dimensiones externas (cm) } & \multirow{2}{*}{ Contenido } \\
\cline { 3 - 4 } & 116 & diámetro mayor & diámetro menor & 2 pichones \\
I & 16 & 9 & y huevos \\
\hline II & 126 & 15 & 10 & vacío \\
\hline III & 110 & 19 & 1 huevo \\
\hline IV & 138 & 20 & 8 & 6 huevos \\
\hline V & 131 & 14 & 12 & vacío \\
\hline VI & 135 & 16 & - & 5 huevos \\
\hline VII & 120 & - & 17 & cascarones \\
\hline VIII & 98 & 23 & 17 & vacío \\
\hline IX & 100 & 19 & 3 huevos \\
\hline
\end{tabular}


Tabla II. Dimensiones de los huevos de Lonchura malacca en una colonia de nueve nidos, localizada en un cañaveral de Quivicán, Cuba, en agosto de 2015

\begin{tabular}{|c|c|c|c|}
\hline Nido & Huevo & Diámetro mayor (mm) & Diámetro menor (mm) \\
\hline III & 1 & 18.1 & 11.4 \\
\hline \multirow{6}{*}{ IV } & 1 & 18.5 & 12.5 \\
\hline & 2 & 19.4 & 12.5 \\
\hline & 3 & 20.5 & 12.4 \\
\hline & 4 & 19.4 & 12.6 \\
\hline & 5 & 18.0 & 12.2 \\
\hline & 6 & 18.9 & 12.4 \\
\hline \multirow{5}{*}{ VI } & 1 & 17.0 & 11.9 \\
\hline & 2 & 17.6 & 11.8 \\
\hline & 3 & 16.6 & 11.8 \\
\hline & 4 & 17.5 & 12.0 \\
\hline & 5 & 17.5 & 11.9 \\
\hline \multirow{3}{*}{ IX } & 1 & 17.9 & 11.5 \\
\hline & 2 & 17.7 & 10.8 \\
\hline & 3 & 18.4 & 11.6 \\
\hline
\end{tabular}

Los nidos II, V, VII y VIII se encontraron sin huevos, y el nido I con tres huevos y dos pichones recién eclosionados. En este último no se tomaron medidas a los huevos.

Todos los nidos se ubicaban en el interior de la parcela y a más de dos metros del borde, lo que hacía difícil su detección. Uno de los nidos estaba depredado, probablemente por ratones (Mus musculus), y contenía cascarones con restos de yema de huevo. Siete días después, la colonia fue extirpada por las actividades agrícolas propias del manejo del campo de caña de azúcar.

Durante la semana de estudio, la zona fue explorada y se comprobó la presencia de dos colonias de nidificación en otras parcelas de caña, de seis y ocho nidos respectivamente. Los cultivos tenían la misma edad y características de la primera plantación. El material vegetal de la estructura de los nidos estaba relativamente fresco y en su interior existían heces y fragmentos de cascarones, lo que sugirió una actividad reciente para ambas colonias. Durante estos recorridos, se detectaron seis bandadas de monjitas, compuestas de adultos y juveniles. El tamaño de dos de estas bandadas superó los cincuenta individuos, y se estimaron en total doscientas aves. 
El uso de campos de caña por parte de la Monjita Tricolor para nidificar ha sido previamente documentado en otras regiones del Caribe (Burger y Gochfeld, 1989; Latta et al., 2006). Según los últimos autores, el tamaño de puesta de la especie usualmente no supera los cinco huevos, lo que coincide con lo encontrado en este estudio.

Dentro de su rango nativo de distribución, L. malacca nidifica en gramíneas cercanas a fuentes de agua. Construye nidos globosos de $17.0 \times 12.0 \mathrm{~cm}$, en los que deposita entre seis y siete huevos de $11.0 \pm 0.3 \mathrm{~mm}$ x $6.0 \pm 0.5 \mathrm{~mm}$ (Singh et al., 2001). En este trabajo, las forma y dimensiones de los nidos fueron semejantes a las registradas; los huevos, en cambio, presentaron un mayor tamaño.

Según las observaciones de Singh et al., (2001) y Payne (2018) la Monjita Tricolor construye el nido en alrededor de una semana y seguidamente comienza a poner huevos en días consecutivos. Inicia la incubación cuando completa la puesta y los pichones eclosionan pasados los 14 días. Basado en estos datos y nuestras observaciones, la actividad reproductiva en la zona de estudio debió iniciar a finales de mayo. Probablemente la colonia activa encontrada estuviera entre las últimas de la temporada. En este sentido, Garrido y Kirkconnel (2011) sugieren que la especie cría en Cuba entre abril y agosto.

El presente trabajo documenta por primera vez para Mayabeque, Cuba, el uso de campos de caña, por parte de L. malacca para nidificar. El impacto de esta especie, ampliamente distribuida en la región, sobre la biología de otras aves granívoras nativas así como en los cultivos agrícolas no ha sido evaluado hasta el momento. Los datos presentados aquí permiten conocer las características de sus colonias reproductivas, así como las fechas más probables de su actividad; lo que puede ser muy útil para futuras acciones de manejo y control.

\section{AGRADECIMIENTOS}

Agradecemos a Duviel Barrios, quien ubicó y facilitó el acceso a la primera colonia de Monjita, y a Kevin A. García y Daylín Pantoja por su apoyo durante el trabajo de campo. A Dennis Denis, Ormaily Madruga y dos revisores anónimos por sus valiosos comentarios al manuscrito inicial.

\section{LITERATURA CITADA}

Acosta, M. y L. Mugica. 2006. Aves en el ecosistema arrocero. Capítulo 6. En: Mugica, L., D. Denis, M. Acosta, A. Jiménez y A. Rodríguez, eds. Aves acuáticas en los humedales de Cuba. Editorial Científico-Técnica, Cuba, 108-135 pp.

Avery, M. L. 1979. Food preferences and damage levels of some avian rice field pests in Malaysia. Bird Control Seminars Proceedings. University of Nebraska, Lincoln, 161-166 pp.

BirdLife International. 2016. Lonchura malacca, The IUCN Red List of Threatened Species. e.T22719837A94647350. Disponible en: http://dx.doi.org/10.2305/IUCN.UK.2016-3. RLTS.T22719837A94647350 (accesado: 11 de marzo de 2016).

Burger, J. y M. Gochfeld. 1989. Nest site selection by the exotic Chestnut Mannikin (Lonchura malacca) in Puerto Rico. Biotropica, 21 (4): 364-368.

De Juana, E. 2006. Observaciones de aves raras en España, 2004. Ardeola, 53 (1): 163-190. 
Garrido, O. H. y A. Kirkconnell. 2011. Aves de Cuba. Cornell University Press, Ithaka, NY, 399 pp.

Kirkconnell, A. y O. H. Garrido. 1992. Lonchura malacca (Aves: Estrildidae), nueva especie para la avifauna cubana. The Journal of Caribbean Ornithology, 5 (1): 2.

Latta, S., C. Rimmer, A. Keith, J. Wiley, H. Raffaele, K. McFarland y E. Fernández. 2006. Birds of the Dominican Republic and Haiti. Princenton University Press, 381 pp.

Lever, C. 2005. Naturalised birds of the World. T y AD Poyser, London, 350 pp.

Otero-Vázquez, J. L. 1998. Riqueza de aves del Cañón de San Cristóbal, Puerto Rico. The Journal of Caribbean Ornithology, 11 (2): 32-35.

Payne, R. B. 2018. Tricolored Munia (Lonchura malacca). Handbook of the Birds of the World Alive, Lynx Edicions. Disponible en : https://www.hbw.com/node/61194 (accesado: 24 de septiembre de 2018).

Raffaele, H., J. W. Wiley, O. Garrido, A. Keith y J. Raffaele. 2003. Birds of the West Indies. Princenton University Press, 216 pp.

Reidinger Jr., R. F. y J. L. Libay. 1979. Perches coated with glue reduce bird damage in ricefield plots. Bird Control Seminars Proceedings. University of Nebraska, Lincoln, 201-206 pp.

Rodríguez, D., E. Ruiz, A. Parada y A. Hernández. 2014. Composición y distribución de las aves. Capítulo 3.3. En: Rodríguez, D., A. Arias y E. Ruiz, eds. Fauna terrestre del Archipiélago de Sabana-Camagüey, Cuba. Editorial Academia, La Habana, 218-261 pp.

Saucedo, O. M., L. E. Fernández, R. Quiñones, G. Rodríguez y A. Moya. 2017. Las aves granívoras y el cultivo del sorgo en la provincia de Villa Clara, Cuba. Revista Centro Agrícola, 44 (2): 36-43.

Sharpe, C., D. Ascanio y R. Restall. 1997. Three species exotic passerine in Venezuela. Cotinga, 7: $43-44$.

Singh, L. A. K., S. S. Srivastava y S. D. Rout. 2001. Observations on nesting ecology of Black-headed Munia (Lonchura malacca malacca). Zoos'Print Journal, 16 (2): 423-427.

[Recibido: 13 de octubre, 2019. Aceptado para publicación: 11 de abril, 2020] 